\title{
Laser Pump-Probe Experiments on Microsecond to Millisecond Timescales at an Electrostatic Ion Storage Ring: Triplet-Triplet Absorption by Protoporphyrin-IX Anions
}

\author{
Kristian Støchkel, Jean Ann Wyer, Maj-Britt Suhr Kirketerp, and \\ Steen Brøndsted Nielsen \\ Department of Physics and Astronomy, Aarhus University, Aarhus, Denmark
}

\begin{abstract}
Here we demonstrate that pump-probe experiments can be carried out on microsecond to millisecond timescales using an electrostatic ion storage ring. As a test case, we have chosen protoporhyrin IX anions that have lifetimes with respect to dissociation after photoexcitation on this time scale. Ions were photoexcited on one side of the ring with either 430- or 535-nm light (pump) and then allowed to take a certain number of revolutions before they were photoexcited by a second laser pulse (probe) with wavelengths between 650 and $950 \mathrm{~nm}$. If ions were first excited by the pump, an increased yield of neutral products caused by the absorption of red light was measured in a microchannel plate detector located on the other side of the ring. This implies that it is possible to pick out ions that were photoexcited by the pump pulse and to spectroscopically characterize these ions. We report absorption spectra of $535 \mathrm{~nm}$ photoexcited porphyrin anions, with time delays of 0.19 and $0.57 \mathrm{~ms}$ between the pump and probe pulses, and find that absorption occurs over a broad region in the red. (J Am Soc Mass Spectrom 2010, 21, 1884-1888) ( 2010 Published by Elsevier Inc. on behalf of American Society for Mass Spectrometry
\end{abstract}

$\mathrm{T}$ ime-resolved experiments on ions in vacuo have been carried out with great success on the femtosecond to picosecond timescales in recent years. On this timescale, the ions only move slightly between the laser pulses, and the experiment therefore relies on a good overlap between the two laser beams for the probe photons to interact with the same molecules that were excited by the pump photons. These experiments have provided important information on lifetimes of electronically excited states and on de-excitation pathways back to the electronic ground state. Examples include protonated aromatic amino acids, peptides, flavin, DNA nucleobases, $\mathrm{C}_{60}$, and cluster anions [1-9].

To study longer timescale processes, larger time delays between the pump and probe laser beams are needed. Dunbar and coworkers [10-12] have investigated the radiative cooling of ions in an ion cyclotron resonance (ICR) ion trap using time delays between the two laser pulses of $100 \mathrm{~ms}$ and up to seconds. On the shorter timescale of microseconds to milliseconds, there is very little work. Using UV light, Joly et al. [13] recently photodetached an electron from a tryptophanbased pentapeptide dianion stored in a quadrupole ion trap. The radical monoanion was shown to be stable for

Address reprint requests to Drs. K. Støchkel or S. Brøndsted Nielsen, Department of Physics and Astronomy, Aarhus University, Ny Munkegade, DK-8000 Aarhus, Denmark. E-mail: krst@phys.au.dk or sbn@phys. au.dk long periods (at least $40 \mu \mathrm{s}$ ) based on photodissociation experiments using a probe laser pulse (visible light) delayed in time.

In the present work, our goal is to address long-lived excited states. For example, photoexcitation of an evenelectron species to a singlet state is often followed by intersystem crossing to a lower-lying triplet state. Such a molecule is trapped in its triplet state for a long time since the required spin-flip introduces a bottleneck for returning to the electronic ground state. Porphyrins are known to have high triplet-quantum yields and tripletstate lifetimes of microseconds to milliseconds [14-18]. Hence they are well-suited as test cases for triplet-triplet absorption spectroscopy. Gas-phase time-resolved experiments at these long timescales are possible using ion traps, e.g., quadrupolar and ICR ion traps. Here we show, using protoporphyrin IX (pp) anions (Figure 1), how the implementation of two pulsed lasers at an electrostatic ion storage ring also allows for such experiments. A benefit of the ring technique is that only ions with the same mass-to-charge ratio are stored at any time.

\section{Experimental}

Experiments were performed at the electrostatic ion storage ring in Aarhus (ELISA) (see Figure 2) [19-21]. Electrospray ionization was used to produce ions, 


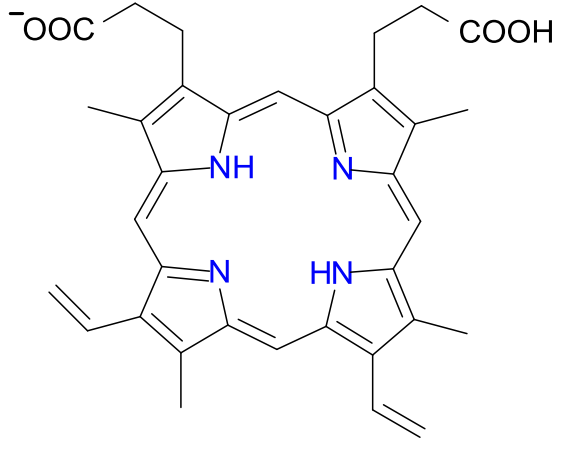

Figure 1. Structure of protoporphyrin IX (pp) anions.

which were subsequently accumulated in a 22-pole ion trap and thermally equilibrated by collisions with a helium buffer gas therein (Figure 2). The ions were accelerated as an ion bunch to kinetic energies of 22 $\mathrm{keV}$, and a bending magnet was used to select the appropriate ions. Following injection into the ring, the ions were stored for about $35 \mathrm{~ms}$ to ensure the decay of highly vibrationally excited ions before being irradiated by a nanosecond light pulse from a tunable laser (Spectra Physics). This is an Nd:YAG laser where the third harmonic (355 $\mathrm{nm}$ ) pumps an optical parametric oscillator (OPO), which subsequently has a visible output. The excitation wavelength for the experiment was chosen to be either 430 or $535 \mathrm{~nm}$. The photoexcited ions were allowed to take a certain number of revolutions before they were excited in the same region as before by a second laser pulse from a tunable laser (EKSPLA). This is also an Nd:YAG laser in combination with an OPO. The wavelength was scanned between 650 and $950 \mathrm{~nm}$. Note that at $709 \mathrm{~nm}$ the beam-exit window of the laser changes. For the 430-nm pump experiment, a dichroic mirror that reflects blue light (pump) and transmits red light (probe) was used to align the beams. For the 535-nm pump experiment, the dichroic mirror does not reflect. Instead, the mirror was removed and a nearly parallel path was introduced for the probe beam relative to the pump beam using two different mirrors. The repetition rate of the experiment was $10 \mathrm{~Hz}$. Lifetimes with respect to dissociation were obtained from measurements of the yield of neutrals at each cycle hitting the microchannel plate (MCP) detector located at the end of the straight section opposite the straight side where photoexcitation was performed (i.e., delayed dissociation).

The pressure in the ring was of the order of $10^{-10}$ mbar, which set an upper limit of $1-2 \mathrm{~s}$ on the storage time. The protoporhyrin IX compound was purchased from Sigma-Aldrich (St. Louis, MO, USA) and dissolved in methanol.

\section{Results and Discussion}

A time spectrum of pp anions is shown in Figure 3. The high count rate after injection is due to the decay of vibrationally excited ions that were formed during the transmission from the 22-pole ion trap to the ring. After
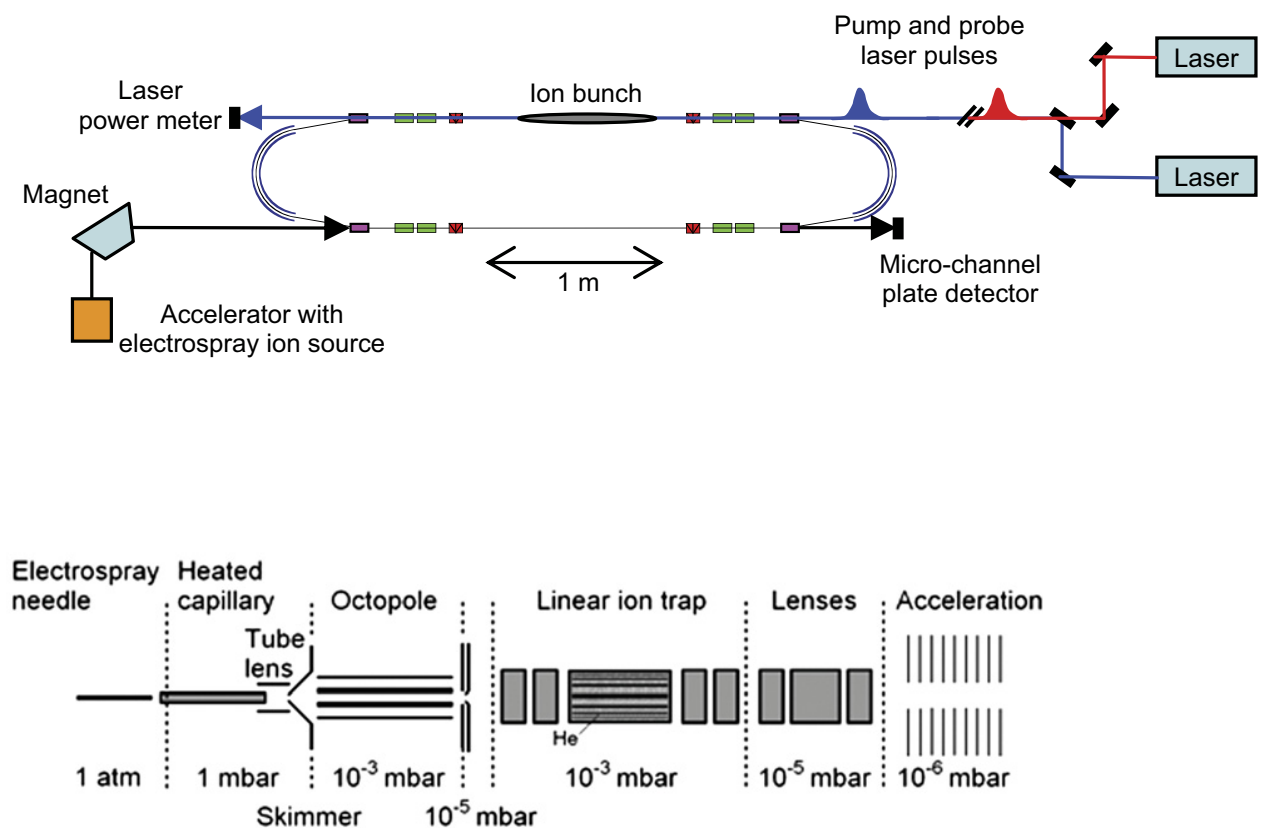

Figure 2. Two-laser experiment at the electrostatic ion storage ring in Aarhus (ELISA). Ions are stored in the ring for a certain number of revolutions before they are photoexcited by the first laser pulse (pump). A second laser pulse (probe) delayed in time relative to the first is then used to monitor photoexcited ions from an increased yield of neutrals in the MCP detector. Note that the delay times chosen correspond to three or more revolutions of the ions in the ring; in other words ions move in space between the two pulses. The ion source including the 22-pole ion trap is also shown. See text for more details. 


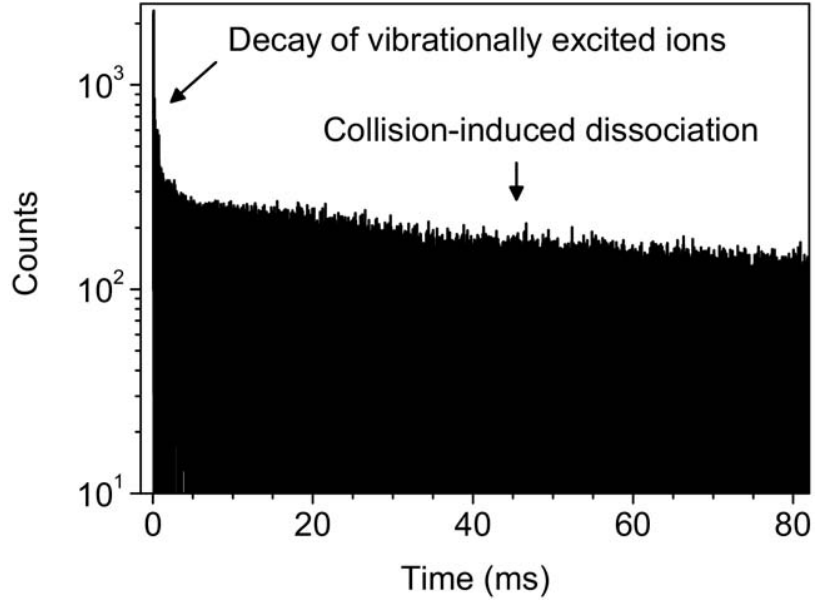

Figure 3. Time spectrum of $\mathrm{pp}$ anions.

about $5 \mathrm{~ms}$, the decay rate is nearly constant and is ascribed to collisions with residual gas in the ring. The time constant for storage of the ions in the ring is $200 \mathrm{~ms}$ according to a long time measurement (Figure S1, which can be found in the electronic version of this article).

After about $35 \mathrm{~ms}$, the ions were irradiated by light $(430 \mathrm{~nm})$ that lies within the Soret absorption band region ( $S_{0} \rightarrow S_{2}$ transition). This gave rise to an increased yield of neutrals as can be seen in the delayed dissociation measurement (see Figure 4a). Hence, the ions absorb light at this wavelength. There is a fast decay process with a time constant of $67 \pm 30 \mu \mathrm{s}$, assigned to statistical dissociation after internal conversion to the electronic ground state, and a slower decay process with a time constant of $0.87 \pm 0.30 \mathrm{~ms}$ (Figure 4a). The slow one is likely due to the trapping of ions in an excited-triplet state that acts as a bottleneck for dissociation as has been discussed in detail earlier [22, 23]. Briefly, intersystem crossing back to the electronic ground state is slow and occurs approximately with the time constant of $0.87 \mathrm{~ms}$. A power-dependence measurement reveals that the long-lifetime component is due to one-photon absorption. The short-lifetime component is dominated by one-photon absorption, with the contribution from two-photon absorption estimated to be less than $10 \%$. Finally, we note that the dominant dissociation channel is loss of $\mathrm{CO}_{2}$ as was earlier reported [22], and that the photon energy is not high enough for photodetachment to occur since the electron binding energy of the anion is at least $3.4 \mathrm{eV}$ (electron binding energy of $\mathrm{CH}_{3} \mathrm{CH}_{2} \mathrm{COO}^{-}$).

To spectroscopically characterize the slowly decaying photoexited ions, we fired a second laser pulse after the ions had taken seven cycles in the ring (revolution time is $95.2 \mu \mathrm{s})$. At this time most of the vibrationally excited ions had decayed (cf., fast decay process). Triplet-state excited protoporphyrins in solution have been reported to absorb above $550 \mathrm{~nm}[15,18]$. We therefore chose a probe wavelength of $684 \mathrm{~nm}$ to be far away from the strong absorption of the ground-state ions themselves. An increased count rate due to absorption of the probe laser light is clearly visible (Figure $4 b$ ). While when the experiment was repeated with the pump laser switched off, no increase in the yield of neutrals was observed (Figure 4c). Possibly, the ions absorb at this wavelength but the photon flux was too low for two-photon absorption to occur (about $1.5 \mathrm{~mJ}$ per pulse, laser beam size diameter of about $2 \mathrm{~mm}$ ). Hence, absorption of 684-nm light is only sufficient to cause dissociation from already photoexcited ions. A similar finding was obtained by pumping with $535-\mathrm{nm}$ light in the Q-band absorption region ( $\mathrm{S}_{0} \rightarrow \mathrm{S}_{1}$ transition).

The results from pump-probe experiments taken with different time delays are shown in Figure 5. There it can be seen that the laser-induced probe signal shifts in time in accordance with the pump-probe delay. Not surprisingly, it is evident that the absorption of the probe light decreased as the time delay increased due to fewer and fewer photoexcited ions.

Next we decided to measure the action spectrum of the 535-nm photoexcited ions. This experiment is com-

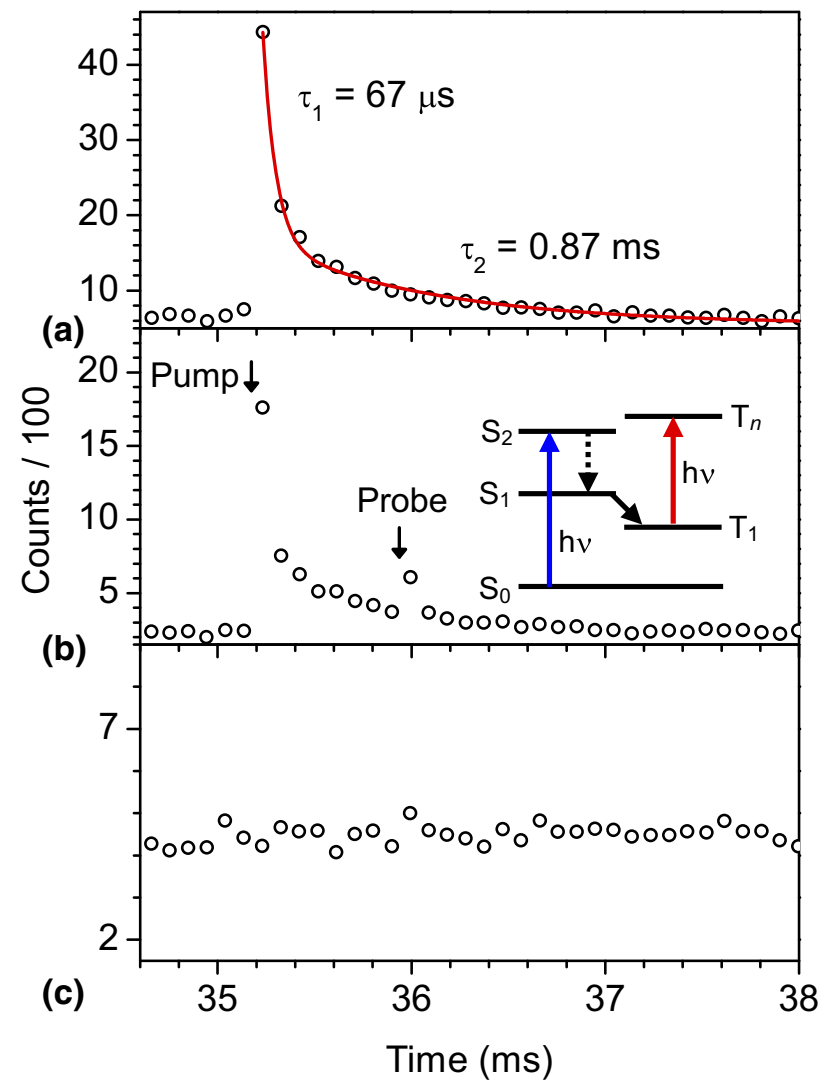

Figure 4. (a) Time spectrum of $\mathrm{pp}$ anions that were photoexcited after 35.19 ms by 430-nm light (pump). (b) Same as (a) but a probe laser pulse $(684 \mathrm{~nm})$ was fired $0.67 \mathrm{~ms}$ after the pump laser pulse. A simplified Jablonski diagram is shown to illustrate the photophysical processes: after excitation to the $\mathrm{S} 2$ state, internal conversion occurs to $\mathrm{S} 1$ followed by intersystem crossing to the $\mathrm{T} 1$ triplet state. Ions in this state absorb red light. (c) Time spectrum of pp anions that were photoexcited by the probe laser $(684 \mathrm{~nm})$ at 35.86 ms. No prior pump pulse was used and, consequently, no absorption was seen after firing the probe laser. 


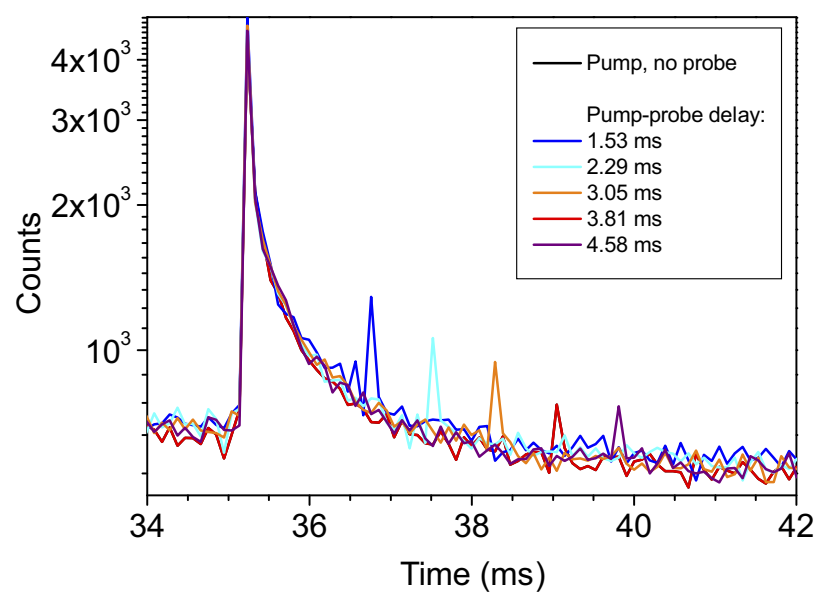

Figure 5. Time spectra of $\mathrm{pp}$ anions that were photoexcited after $35.19 \mathrm{~ms}$ by $535-\mathrm{nm}$ light. In each spectrum the probe laser pulse $(660 \mathrm{~nm})$ was fired after different delay times. The laser-induced probe signal shifts in accordance with the pump-probe delay.

plicated by the fact that fluctuations in the two laser beam intensities need to be corrected. However, this is simplified as variations in the pump laser power were less than $5 \%$. Therefore, in the analysis the power of the pump laser was taken to be constant for all measurements. A correction for variations in ion beam intensity is also needed and was done based on the yield of neutrals before photoexcitation. This yield is the result of collisions between ions and residual gas and is therefore directly proportional to the number of ions in the ring. The increased yield of neutrals due to the probe laser represents the absorption by the photoexcited ions (i.e., action spectroscopy). After correcting for ion beam fluctuations, the yield at each wavelength was subsequently divided by the number of photons in the probe laser beam. The number of photons in the probe laser beam was obtained from an established correlation between wavelength and laser output power and is estimated to be associated with an uncertainty of $10 \%$. We estimate that the overall uncertainty in the yield of photoneutrals is about $20 \%$. This high uncertainty makes it difficult to find the actual positions of band maxima. Nevertheless, action spectra were acquired for the case where the time delay between the pump and probe laser beams was $0.19 \mathrm{~ms}$ and that where it was $0.57 \mathrm{~ms}$ (Figure 6). In both spectra, it can be seen that absorption is strong at $650 \mathrm{~nm}$ and that it decreases as the wavelength increases, with little or no absorption seen above $800 \mathrm{~nm}$. Furthermore, the band appears sharper after the longer delay time $(0.57 \mathrm{~ms})$ than after the shorter one $(0.19 \mathrm{~ms})$, which may be due to a colder population at longer delay times. It would have been useful to go to lower probe wavelengths but this is complicated due to absorption by these anions in their ground state.

The absorption observed here is within the expected absorption region for porphyrins excited to triplet states $[15,18]$. In benzene solution, Chantrell et al. [18] found a very small band centered around $700 \mathrm{~nm}$ for protoporhyrin-IX dimethyl ester. A redshift from gas phase to solution phase is commonly seen for $\pi \pi^{*}$ transitions since the polarizability of a molecule increases with its level of excitation, which favors solutesolvent interactions. It should be stressed that absorption by vibrationally hot $\mathrm{pp}$ anions may also contribute to some of the absorption seen in this region. However, no increase in the yield of neutrals was observed in a separate experiment where irradiation by $685-\mathrm{nm}$ light was carried out right after the injection of ions into the ring (data not shown). Here, the count rate of neutrals is already particularly high (first $5 \mathrm{~ms}$, see Figure 3 ) due to the decay of vibrationally excited ions. These ions are present in the bunch since some collisions with residual gas occur during transfer from the 22-pole ion trap to the ring. From the absence of an increase in neutrals after light irradiation, we conclude that "hot" band absorption is not significant and, therefore, the photoexcited signal due to the probe laser pulse in the pump-probe experiment arises from absorption by triplet-state ions.

In conclusion, we have demonstrated that pumpprobe experiments on the microsecond to millisecond timescale are indeed possible using an electrostatic ion

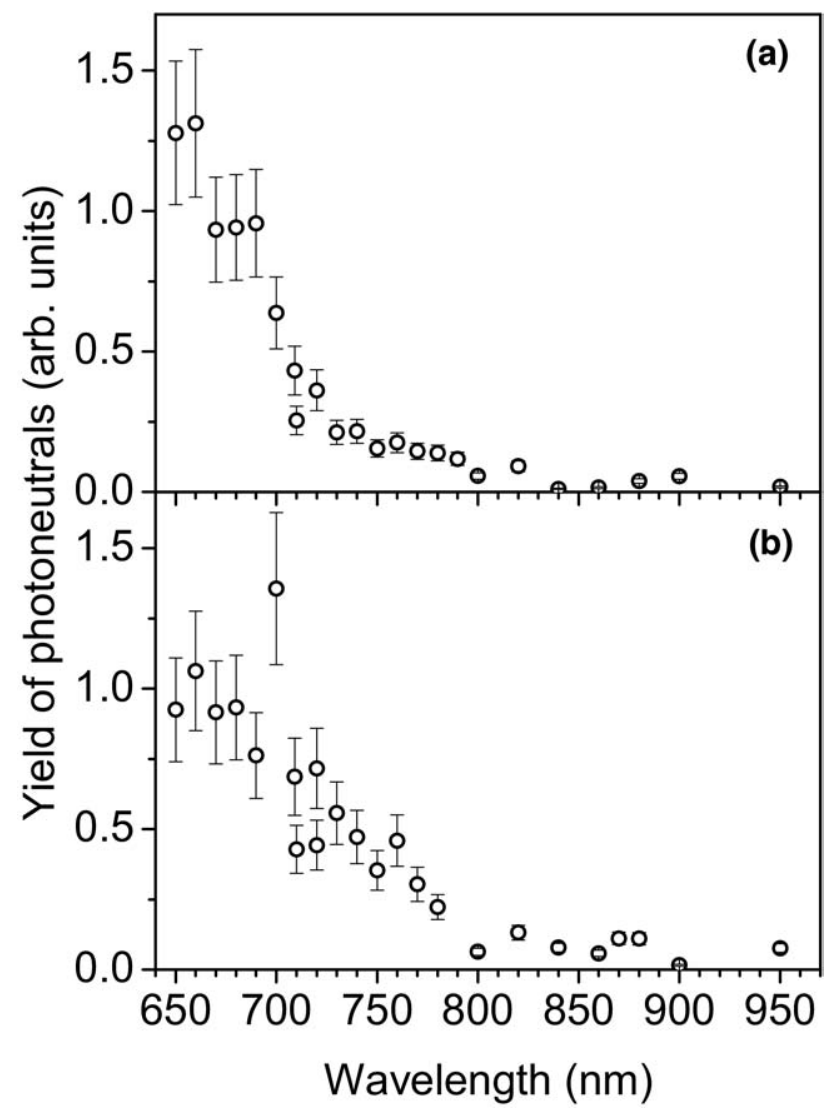

Figure 6. Action spectra obtained as the yield of photoneutrals due to the probe laser pulse as a function of probe wavelength. The pump laser was set to produce light at $535 \mathrm{~nm}$. Time delays between the pump and probe were $0.57 \mathrm{~ms}$ (a) and $0.19 \mathrm{~ms}(\mathbf{b})$. 
storage ring in combination with two lasers. The time resolution of the experiment is the ion revolution time. Finally, we note that ion trap experiments have an advantage over the current setup due to better overlap between the two laser beams and the ion cloud.

\section{Acknowledgments}

The authors acknowledge generous support from Lundbeckfonden, Villum Kann Rasmussen Fonden, and Dir. Ib Henriksens Fond.

\section{Appendix A Supplementary Material}

Supplementary material associated with this article may be found in the online version at doi:10.1016/ j.jasms.2010.07.004.

\section{References}

1. Kang, H.; Jouvet, C.; Dedonder-Lardeux, C.; Martrenchard, S.; Grégoire, G.; Desfrancois, C.; Schermann, J. P.; Barat, M.; Fayeton, J. A. Ultrafast Deactivation Mechanisms of Protonated Aromatic Amino Acids Following UV Excitation. Phys. Chem. Chem. Phys. 2005, 7, 394-398.

2. Grégoire, G.; Kang, H.; Dedonder-Lardeux, C.; Desfrancois, C.; Onidas, D.; Lepere, V.; Fayeton, J. A. Statistical vs. Non-Statistical Deactivation Pathways in the UV Photo-Fragmentation of Protonated TryptophanLeucine Dipeptide. Phys. Chem., Chem. Phys. 2006, 8, 122-128.

3. Nolting, D.; Schultz, T.; Hertel, I. V.; Weinkauf, R. Excited State Dynamics and Fragmentation Channels of the Protonated Dipeptide $\mathrm{H}_{2} \mathrm{~N}-\mathrm{Leu}-\mathrm{Trp}-\mathrm{COOH}$. Phys. Chem., Chem. Phys. 2006, 8, 5247-5254.

4. Guyon, L.; Tabarin, T.; Thuillier, B.; Antoine, R.; Broyer, M.; Boutou, V.; Wolf, J.-P.; Dugourd, P. Femtosecond Pump-Probe Experiments on Trapped Flavin: Optical Control of Dissociation. J. Chem. Phys. 2008, 128, 075103.

5. Nolting, D.; Weinkauf, R.; Hertel, I. V.; Schultz, T. Excited-State Relaxation of Protonated Adenine. Chem. Phys. Chem. 2007, 8, 751-755.

6. Ehrler, O. T.; Yang, J. P.; Hättig, C.; Unterreiner, A. N.; Hippler, H.; Kappes, M. M. Femtosecond Pump/Probe Photoelectron Spectroscopy of Isolated $C_{60}$ Negative Ions. J. Chem. Phys. 2006, 125, 074312.

7. Gerhardt, P.; Niemitz, M.; Kim, Y. D.; Ganteför, G. Fast Electron Dynamics in Small Aluminum Clusters: Non-Magic Behavior of a Magic Cluster. Chem. Phys. Lett. 2003, 382, 454-459.
8. Niemietz, M.; Gerhardt, P.; Ganteför, G.; Kim, Y. D. Relaxation Dynamics of the $\mathrm{Au}_{3}{ }^{-}$and $\mathrm{Au}_{6}{ }^{-}$Cluster Anions. Chem. Phys. Lett. 2003, 380, 99-104.

9. Kim, Y. D.; Niemietz, M.; Gerhardt, P.; von Gynz-Rekowski, F.; Ganteför, G. Relaxation Dynamics of Magic Clusters. Phys. Rev. B 2004, 70, 035421.

10. Dunbar, R. C.; Chen, J.-H. Two-Pulse Photodissociation Measurement of Infrared Fluorescence Relaxation in Bromobenzene Ion. J. Phys. Chem. 1984, 88, 1401-1404.

11. Asamato, B.; Dunbar, R. C. Observation of the Infrared Relaxation of Iodobenzene Ions Using Two-Light-Pulse Photodissociation. J. Phys. Chem. 1987, 91, 2804-2807.

12. Faulk, J. D.; Dunbar, R. C. Ion Cyclotron Resonance Ion Trap Measurements of Energy Relaxation in Gas-Phase Ions: Three Techniques Compared for Thiophenol Ion. J. Phys. Chem. 1989, 93, 7785-7789.

13. Joly, L.; Antoine, R.; Allouche, A.-R.; Dugourd, P. Formation and Spectroscopy of a Tryptophan Radical Containing Peptide in the Gas Phase. J. Am. Chem. Soc. 2008, 130, 13832-13833.

14. Sinclair, R. S.; Tait, D.; Truscott, T. G. Triplet States of Protoporphyrin IX and Protoporphyrin IX Dimethyl Ester. J. C. S. Faraday. I 1980, 76, 417-425.

15. Bonnett, R.; Charalambides, A. A.; Land, E. J.; Sinclair, R. S.; Tait, D.; Truscott, T. G. Triplet States of Porphyrin Esters J. C. S. Faraday. I 1980, $76,852-859$.

16. Lafferty, J.; Truscott, T. G. Triplet State of Protoporphyrin IX. J. C. S. Chem. Commun. 1978, 51-52.

17. Charlesworth, P.; Truscott, T. G.; Kessel, D.; Medforth, C. J.; Smith K. M. Photophysical Studies of Substituted Porphyrins. J. Chem. Soc. Faraday Trans. 1994, 90, 1073-1076.

18. Chantrell, S. J.; McAuliffe, C. A.; Munn, R. W.; Pratt, A. C.; Land, E. J. Excited States of Protoporphyrin IX Dimethyl Ester: Reaction on the Triplet with Carotenoids. J. Chem. Soc. Faraday Trans. 1977, 73, 858-865.

19. Møller, S. P. ELISA, an electrostatic storage ring for atomic physics. Nucl. Instrum. Methods A 1997, 394, 281-286.

20. Andersen, J. U.; Hvelplund, P.; Brøndsted Nielsen, S.; Tomita, S. Wahlgreen, H.; Møller, S. P.; Pedersen, U. V.; Forster, J. S.; Jørgensen, T. J. D. The Combination of an Electrospray Ion Source and an Electrostatic Storage Ring for Lifetime and Spectroscopy Experiments on Biomolecules. Rev. Sci. Instrum. 2002, 73, 1284-1287.

21. Støchkel, K.; Kadhane, U.; Andersen, J. U.; Holm, A. I. S.; Hvelplund, P. Kirketerp, M.-B. S.; Larsen, M. K.; Lykkegaard, M. K.; Brøndsted Nielsen, S.; Panja, S.; Zettergren, H. A New Technique for TimeResolved Daughter Ion Mass Spectrometry on the Microsecond to Millisecond Time Scale Using an Electrostatic Ion Storage Ring. Rev. Sci. Instrum. 2008, 79, 023107.

22. Calvo, M. R.; Andersen, J. U.; Hvelplund, P.; Brøndsted Nielsen, S.; Pedersen, U. V.: Rangama, J.; Tomita, S. Photophysics of Protoporphyrin Ions In Vacuo: Triplet-State Lifetimes and Quantum Yields. J. Chem. Phys. 2004, 120, 5067-5072.

23. Nielsen, C. B.; Forster, J. S.; Ogilby, P. R.; Brøndsted Nielsen, S. Delayed Dissociation of Photoexcited Porphyrin Cations in a Storage Ring. Determination of Triplet Quantum Yields. J. Phys. Chem. A 2005, 109, 3875-3879. 